\title{
ASMA
}

\section{Tiotropio en asma mal controlada con terapia combinada estándar}

\section{Tiotropium in asthma poorly controlled with standard combination therapy}

Diana Cano, MD. (1), Audrey PiotrostanalzkI V., MD. ${ }^{(2)}$

${ }^{(1)}$ Fellow de primer año de Neumología, Universidad de La Sabana, Fundación Neumológica Colombiana. Bogotá, Colombia.

(2)Internista, Neumólogo, Universidad Militar Nueva Granada, Fundación Neumológica Colombiana, Fundación Cardioinfantil. Bogotá, Colombia.

Correspondencia: Audrey Piotrostanalzki. Correo electrónico: apiotrostanalzki@ neumologica.org

Recibido: 4-06-2013. Aceptado: 12-12-2013

\section{Referencia}

Kerstjens HA, Engel M, Dahl R, Paggiaro P, Beck E, Vandewalker $\mathrm{M}$, et al. Tiotropium in asthma poorly controlled with standard combination therapy. N Engl J Med. 2012;367:1198-207.

\section{Pregunta}

En pacientes con asma mal controlada a pesar del uso de una combinación de glucocorticoide inhalado con beta agonista de acción prolongada, ¿es la terapia combinada con tiotropio, en comparación con dicha terapia estándar, eficaz y segura evaluada por pruebas de función pulmonar, mortalidad y frecuencia de exacerbación, en un periodo de 48 semanas?

\section{Diseño}

Dos estudios de asignación aleatoria, doble ciego, controlado con placebo, diseño de grupo paralelo. PrimoTinA-Asthmal y PrimoTinAAsthma2 (referidos como estudio 1 y estudio 2).

\section{Cegamiento}

Pacientes y clínicos evaluadores.

\section{Duración de la intervención}

Cuarenta y ocho semanas semanas. Periodo de inclusión entre octubre de 2008 y julio de 2011.

\section{Lugar}

Estudio multicéntrico en quince países:

- Estudio 1: Australia, Canadá, Dinamarca, Gran Bretaña, Alemania, Italia, Japón, Países Bajos, Serbia, Suráfrica, Taiwán, Turquía, Ucrania, Estados Unidos.

- Estudio 2: Australia, Dinamarca, Gran Bretaña, Alemania, Italia, Japón, Países Bajos, Nueva Zelanda, Serbia, Suráfrica, Taiwán, Turquía.

\section{Pacientes}

\section{Criterios de inclusión}

- Edad 18-75 años. Antecedente de asma mayor a cinco años de evolución y diagnosticado antes de los 40 años.

- Puntaje en el cuestionario de control de asma (Asthma Control Questionaire 7-ACQ-7) mayor o igual a 1,5. 
- Limitación del flujo aéreo persistente definida como un VEF1 postbroncodilatador $\leq 80 \%$ del predicho y capacidad vital forzada $\leq 70 \%$ después de 30 minutos de ser administrados: cuatro aspiraciones de $100 \mathrm{mcg}$ de salbutamol o $90 \mathrm{mcg}$ de albuterol en la visita de tamizaje, a pesar de la terapia diaria con glucocorticoides inhalados $(\geq$ $800 \mathrm{mcg}$ de budesonida o su equivalente) y un LABA.

- Al menos una exacerbación tratada con corticoides sistémicos en el último año.

\section{Criterios de exclusión}

- Fumadores o exfumadores.

- Antecedente de EPOC.

- Enfermedad seria coexistente.

- Uso actual de anticolinérgicos.

\section{Intervención}

- Dos aspiraciones de 2,5 mcg (5 mcg) de tiotropio Respimat ${ }^{\circledR}$ cada mañana, adicional a terapia individual de mantenimiento de asma (alta dosis de corticoide inhalado y LABA).

- Dos aspiraciones de placebo Respimat® cada mañana, adicional terapia individual de mantenimiento de asma (alta dosis de corticoide inhalado y LABA).

Medicamentos permitidos: teofilina, modificadores de leucotrienos, anticuerpos anti IgE y glucocorticoides orales $(\leq 5 \mathrm{mg} /$ día) si la dosis permaneció estable por al menos cuatro semanas antes de estar al estudio.

Medicación de rescate: salbutamol (100 mcg/inhalación) o albuterol (90mcg/inhalación).

\section{Resultados}

De 1.135 pacientes iniciales, 912 pacientes fueron elegibles para la aleatorización. De éstos, 409 pacientes (237 pacientes en el estudio 1 y 219 pacientes en el estudio 2) tiotropio y 405 pacientes (222 pacientes en el estudio 1 y 234 pacientes en el estudio 2) recibieron placebo.

\section{Desenlaces primarios}

- Función pulmonar: en 24 semanas, el promedio de cambio del VEF1 pico medido tres horas posterior a la intervención fue mayor en el grupo de tiotropio que con placebo en los dos estudios: una diferencia de $86 \pm 34 \mathrm{~mL}$ en el estudio $1(\mathrm{p}=0,01)$ y $154 \pm 32 \mathrm{~mL}$ en el estudio $2(\mathrm{p}<0,01)$.

- Exacerbaciones severas: comparado con placebo, el uso de tiotropio incrementó el tiempo transcurrido hasta la aparición de la primera exacerbación grave (282 vs. 226 días, reducción global de riesgo del 21\% -HR: 0,79; $p=0,03-$, NNT para prevenir una exacerbación durante 48 semanas de tratamiento de 15 .

\section{Desenlaces secundarios}

- Medición de $V_{E F}$ pico y CVF en cada visita del estudio: mejoraron las variables espirométricas en los pacientes del grupo de tiotropio comparadas con el placebo.

- La mediana del tiempo hasta la primera exacerbación del asma, medido como el primer momento de deterioro de los síntomas diarios o descenso $\geq 30 \%$ del pico flujo espiratorio matutino por dos días consecutivos fue mayor en el grupo de tiotropio (315 vs. 181 días), con reducción del riesgo del 31\% (HR 0,69; IC 95\% 0,58-0,82; p<0,001).

- La medición de síntomas y la calidad de vida al paso de los días durante las 48 semanas a través de Cuestionario de Control de Síntomas del Asma y Calidad de Vida ACQ-7 y AQLQ muestra tendencia a la mejoría en el grupo de tiotropio en el estudio 2, aunque en el estudio 1 la diferencia en ALQL no fue significativa.

- Uso de medicación de rescate

- No se encontraron diferencias significativas en los dos grupos.

Eventos adversos: se reportaron $73,5 \%$ en el grupo de tiotropio y $80,3 \%$ en el placebo; no hubo muertes. La presencia de rinitis alérgica y boca seca fue mayor en el grupo de tiotropio. Se reportaron eventos adversos en 77 pacientes: $37(8,1 \%)$ en el grupo tiotropio y $40(8,8 \%)$ en el placebo. En el grupo del tiotropio dos pacientes presentaron exacerbación de 
asma severa y un paciente un evento cerebrovascular. Ucurrieron eventos cardiovasculares en menos del $2 \%$ de los pacientes, los cuales se distribuyeron igual en los dos grupos.

\section{Conclusión}

En pacientes con asma mal controlada a pesar del tratamiento de mantenimiento con glucocorticoides inhalados y LABA, que no tengan reversibilidad en la espirometria posterior a uso de beta 2 agonistas, la adición de tiotropio mostró un efecto significativo de broncodilatación sostenida y aumentó significativamente el tiempo transcurrido hasta la aparición de una primera exacerbación severa.

\section{Comentario}

Entre el 5\% y el 10\% de los pacientes con diagnóstico de asma son de difícil control $(1,2)$, con opciones terapéuticas limitadas. Los resultados presentados por este artículo muestran que la adición de tiotropio a través del dispositivo de inhalación Respimat $^{\circledR}(4)$ se asoció con aumento del tiempo transcurrido hasta la aparición de la primera exacerbación en relación con aquellos a quienes se adicionó placebo; se resalta que el grupo seleccionado para ingreso al estudio presentaba la característica de no tener un cambio espirométrico significativo en el VEF1 posterior a la administración de beta agonista, siendo posible que fueran pacientes con remodelación asociada a asma. No se incluyeron pacientes con enfermedad cardiaca, por lo tanto, la administración de esta terapia debe realizarse con precauciones. Se continúa aún a la espera de estudios de seguridad en asma; ya hay publicados en EPOC (6).

Otros autores han encontrado resultados similares; Peters y colaboradores (5) documentaron en pacientes con asma leve a moderada que la adición de tiotropio a monoterapia con glucocorticoides inhalados, fue igual de efectiva a la adición de salmeterol, pero más efectiva que doblar la dosis de corticoides inhalados. Kerstjens y colaboradores han realizado varias publicaciones al respecto (4). Se considera tanto un mecanismo broncodilatador sinérgico al uso de beta agonistas (2), como un efecto directo antiinflamatorio en la vía aérea, disminuyendo la inflamación eosinofílica (3).

En general, el análisis del presente estudio permite concluir que en pacientes con asma de difícil control a pesar del manejo óptimo con corticoides inhalados y LABA, con persistencia en limitación fija al flujo de aire, la adición de tiotropio disminuye el riesgo de exacerbaciones; la seguridad en la administración de esta terapia requiere mayor evidencia.

\section{Conflictos de intereses}

Los autores declaran no tener conflictos de interés.

\section{Bibliiografía}

1. Bousquet J, Mantzouranis E, Cruz AA, et al. Uniform definition of asthma severity, control, and exacerbations: document presented for the World Health Organization Consultation on Severe Asthma. J Allergy Clin Immunol. 2010;126:926-38.

2. Antoniu SA, Antohe I. Evaluation of inhaled tiotropium in asthma, uncontrolled with standard combination therapy. Expert Opin Pharmacother. 2013;14;967-969.

3. Buels KS, Jacoby DB, Fryer AD. Non-bronchodilating mechanisms of tiotropium prevent airway hyperreactivity in a guinea-pig model of allergic asthma. Br J Pharmacol. 2012;165:1501-14.

4. Kerstjens HA, Disse B, Schröder-Babo W, et al. Tiotropium improves lung function in patients with severe uncontrolled asthma: a randomized controlled trial. J Allergy Clin Immunol. 2011;128:308-14.

5. Peters SP, Kunselman SJ, Icitovic N, et al. Tiotropium bromide step-up therapy for adults with uncontrolled asthma. N Engl J Med. 2010;363:1715-26.

6. Wise R, Anzueto A, Calverley P, Dahl R, Dusser D, Pledger G, et al. The Tiotropium Safety and Performance in Respimat Trial (TIOSPIR), a large scale, randomized, controlled, parallel group trial design and rationale. Respira Res. 2013;14:40. 